\title{
Surface-Acoustic-Wave-Induced Transport in a Double Quantum Dot
}

\author{
W. J. M. Naber, ${ }^{1,2,3}$ T. Fujisawa, ${ }^{2,4}$ H. W. Liu, ${ }^{2,5}$ and W. G. van der Wiel ${ }^{1,6}$ \\ ${ }^{1}$ SRO NanoElectronics, MESA ${ }^{+}$Institute for NanoTechnology, University of Twente, \\ P.O. Box 217, 7500 AE Enschede, The Netherlands \\ ${ }^{2}$ NTT Basic Research Laboratories, NTT Corporation, 3-1 Morinosato-Wakamiya, Atsugi, Kanagawa 243-0198, Japan \\ ${ }^{3}$ Kavli Institute of NanoScience, Delft University of Technology, P.O. Box 5046, 2600 GA Delft, The Netherlands \\ ${ }^{4}$ Tokyo Institute of Technology, 2-12-1 Okayama, Meguro-ku, Tokyo 152-8551, Japan \\ ${ }^{5}$ SORST-JST, 4-1-8 Honmachi, Kawaguchi, Saitama 331-0012, Japan; \\ National Laboratory of Superhard Materials, Institute of Atomic and Molecular Physics, Jilin University, Changchun 130012, China \\ ${ }^{6}$ PRESTO-JST, 7-3-1, University of Tokyo, Hongo, Bunkyo-ku, Tokyo 113-8656, Japan
}

(Received 9 January 2006; published 7 April 2006)

\begin{abstract}
We report on nonadiabatic transport through a double quantum dot under irradiation of surface acoustic waves generated on chip. At low excitation powers, absorption and emission of single and multiple phonons are observed. At higher power, sequential phonon assisted tunneling processes excite the double dot in a highly nonequilibrium state. The present system is attractive for studying electron-phonon interaction with piezoelectric coupling.
\end{abstract}

DOI: 10.1103/PhysRevLett.96.136807

PACS numbers: 73.23.Hk, 63.20.Kr, 77.65.Dq

Electron-phonon coupling often leads to dissipation and decoherence problems in nanoelectronic devices. The decoherence in a tunable two-level quantum system (qubit), such as a double quantum dot (DQD) [1], is of particular interest in the recent light of quantum computation and information [2]. It was found that piezoelectric coupling to acoustic phonons is the dominant mechanism for inelastic transition between two charge states in a DQD [3], as confirmed by theory [4]. In analogy to quantum states in natural atoms - which dominantly couple to and are successfully controlled by photons - the electronic states in solid state systems may be controlled by phonons, taking advantage of the strong electron-phonon coupling.

Because of the piezoelectric coupling in GaAs, surface acoustic waves (SAWs) can be generated by applying a microwave signal to an interdigital transducer (IDT) [5]. The accompanying propagating and oscillating potential has been used in several experiments to transport photogenerated electrons and holes in so-called "dynamical quantum dots" [6]. In those experiments, however, the SAWs give rise to an adiabatic change of the electronic states, where the carriers remain in an eigenstate of the temporal potential.

In this Letter, we present nonadiabatic transitions in a lithographically defined DQD under irradiation of coherent SAWs. We observe resonant phonon assisted tunneling, where transport is well described by considering absorption and emission of one or multiple phonons during the tunneling process [7]. The present results unambiguously indicate a finite contribution of SAWs to the bosonic environment of a quantum two-level system formed by a DQD. Moreover, these transport measurements allow us to determine extremely small amplitudes of the local piezoelectric potential.

Figure 1(a) is a picture of our device showing the $\mathrm{Ti} / \mathrm{Au}$ gate patterns of the interdigital transducer used for gener- ating SAWs on the left and the DQD on the right on top of a GaAs/AlGaAs heterostructure with a 2D electron gas (2DEG) $100 \mathrm{~nm}$ below the surface. The periodicity of the IDT is $1.4 \mu \mathrm{m}$, setting the SAW wavelength $\lambda_{\mathrm{SAW}}$ and
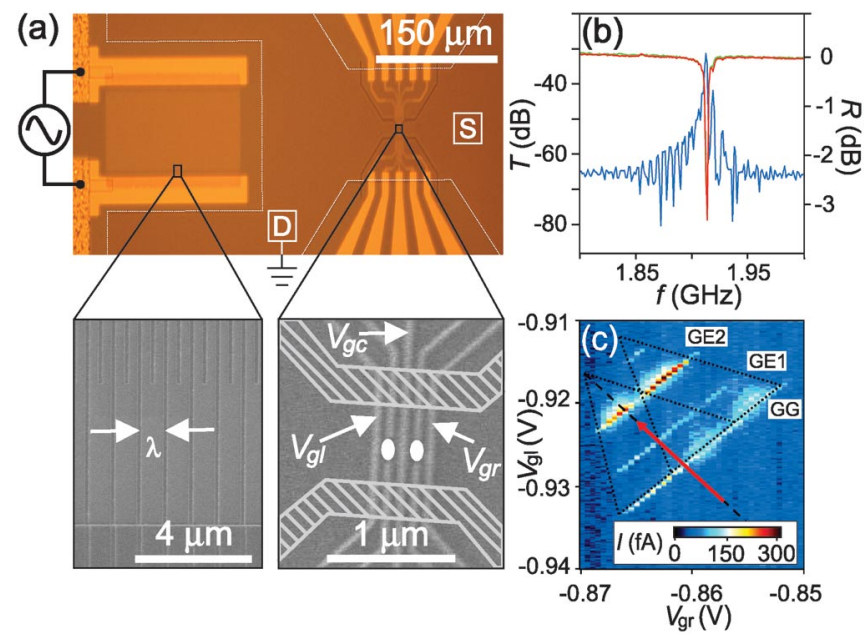

FIG. 1 (color). (a) Picture of the device with an IDT (left) and a DQD (right). The source (S) and drain (D) reservoirs are indicated. The IDT-DQD distance is $227.5 \mu \mathrm{m}$. In the SEM of the IDT, the electrodes, separated by $\lambda=1.4 \mu \mathrm{m}$, are visible. In the hatched regions of the DQD SEM, the 2DEG is depleted by shallow dry etching. The position of the dots is indicated by white dots. (b) Transmission $T$ (blue curve) and reflection $R$ (red and green curves) at room temperature of two IDTs similar to the one used in the experiments, separated by a distance of $455 \mu \mathrm{m}$. A clear peak in $T$ and a dip in $R$ are visible at $1.92 \mathrm{GHz}$. (c) Color scale plot of the DQD current as a function of gate voltages $V_{\mathrm{gl}}$ and $V_{\mathrm{gr}}$ at source drain voltage $V_{\mathrm{SD}}=500 \mu \mathrm{V}$ without SAW generation. The conductance triangles are accentuated by dotted lines. Resonant tunneling lines are clearly visible. The dual gate sweep direction for the SAW experiments is indicated by the red arrow. 
corresponding to a SAW frequency of about $2 \mathrm{GHz}$ in GaAs [see lower left scanning electron micrograph (SEM) in Fig. 1(a)]. The IDT design is characterized at room temperature using a different GaAs/AlGaAs heterostructure with two identical IDTs facing each other, allowing for a two-channel microwave measurement. The transmission and reflection spectra in Fig. 1(b) show a clear resonance at $1.92 \mathrm{GHz}$, as expected from the IDT design. The reflection dip is more than $3 \mathrm{~dB}$, indicating that more than half of the incident power is absorbed in the IDT. The transmission reaches a maximum of $-30 \mathrm{~dB}$ at resonance, implying additional loss in the device. Possible mechanisms for power loss are impedance mismatch, electromechanical conversion loss, and Bragg reflection within the IDT. We found that the reflection and transmission spectra do not change when a DQD device is fabricated in the middle between the IDTs. By assuming identical characteristics for both IDTs, acoustic power at the site of the DQD is $15 \mathrm{~dB}$ less than the incident microwave power $P$.

The DQD is formed in an etched channel of $600 \mathrm{~nm}$ width [see hatched dry etching regions in the lower right SEM in Fig. 1(a)] with appropriate voltages to the indicated gate electrodes, which have a $220 \mathrm{~nm}$ spacing [1].

All measurements described below are performed in a dilution refrigerator with a base temperature of $50 \mathrm{mK}$. We have obtained similar results in two different samples, measured in different cryostats. The data shown here are taken from one sample. Each dot contains $\sim 10$ electrons and has a charging energy of $\sim 2 \mathrm{meV}$ and a discrete energy level spacing of $\sim 150 \mu \mathrm{eV}$. The interdot electrostatic coupling is $\sim 200 \mu \mathrm{eV}$, and the tunneling coupling is weak $(\ll 10 \mu \mathrm{eV})$ so that delocalization of states can be neglected. This weak coupling regime is suitable for studying electron-phonon interaction [3].

Figure 1(c) shows the single-electron tunneling current through the DQD versus gate voltages $V_{\mathrm{gl}}$ and $V_{\mathrm{gr}}$ with a large bias voltage of $500 \mu \mathrm{V}$ with no microwave power $(P=0)$ applied to the IDT. The lower and upper (partly overlapping) triangular conduction regions correspond to electronlike and holelike transport through the DQD, respectively [1]. Resonant tunneling through the ground states (GSs) of the two dots corresponds to the current peak at the base of the triangles (labeled GG), while other resonant tunneling between the left GS and the first and second excited states (ESs) of the right dot are also observed (labeled GE1 and GE2). In the following measurements, we simultaneously sweep $V_{\mathrm{gl}}$ and $V_{\mathrm{gr}}$ along the red arrow in Fig. 1(c), so that the energy difference $\Delta E=$ $E_{1}-E_{2}$ between the GS energies of the left dot $\left(E_{1}\right)$ and the right $\operatorname{dot}\left(E_{2}\right)$ is varied. We observe a symmetric current profile around $\Delta E=0$ representing elastic current through the DQD, while inelastic current at $\Delta E>0$ associated with spontaneous emission of phonons is very small in the present experiment.

When microwaves are applied to the IDT, we observe significant broadening and splitting of the resonant tunnel- ing peaks only at the IDT resonant frequency, $f_{\mathrm{SAW}}=$ $1.9446 \mathrm{GHz}$, as seen in the frequency dependence of the current spectrum in Fig. 2(a). The resonance frequency corresponds very well to that of the GaAs reference sample $(1.92 \mathrm{GHz})$ in Fig. 1(b), where the slight deviation is ascribed to the different heterostructure and the lower temperature in the actual device. This good correspondence rules out photon assisted tunneling [8]. There is no reason why there should be an electromagnetic resonance coinciding with the IDT resonance frequency. We also exclude resonant heating, since the energy levels are well separated from the Fermi levels of the leads. The harmonic oscillation of the energy levels as described below cannot be explained in terms of heating either. Note that no broadening is observed at off-resonant frequencies, also indicating that heating and spurious electromagnetic coupling are negligible.

We now look in more detail at the mechanism of the SAW-induced current in Fig. 2(a). The traveling SAW causes a time-dependent potential $V_{\mathrm{ac}} \cos \left(2 \pi f_{\mathrm{SAW}} t\right)$ between the two quantum dots, due to the piezoelectric and deformation coupling. For GaAs at this frequency, the piezoelectric effect is dominant and the deformation coupling can be neglected [9]. As the lithographical dot-dot distance is $d=220 \mathrm{~nm}$ and the SAW wavelength is $\lambda_{\mathrm{SAW}}=1.4 \mu \mathrm{m}, V_{\mathrm{ac}}$ is a fraction of the amplitude of the piezoelectric potential $V_{\text {pe }}, V_{\text {ac }}=\eta V_{\text {pe }}$, where $\eta=$ $\sin \left(\pi d / \lambda_{\mathrm{SAW}}\right) \approx 0.47$. The time-dependent level spacing $\Delta \tilde{E}(t)$ is, therefore, $\Delta E+\eta V_{\mathrm{pe}} \cos \left(2 \pi f_{\mathrm{SAW}} t\right)$. The peak splitting at resonance frequency in Fig. 2(a) can then be

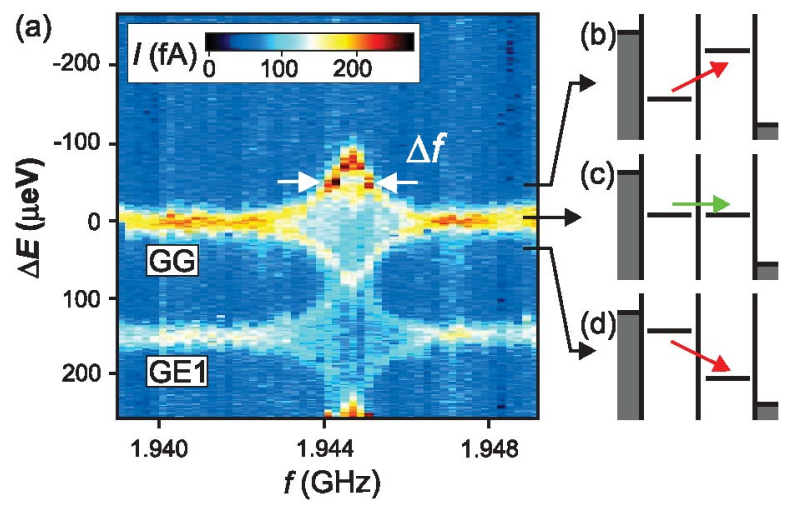

FIG. 2 (color). (a) Color scale plot of the DQD current versus ground state level spacing $\Delta E$ and microwave frequency $f$ applied to the IDT ( $-40 \mathrm{dBm}$ microwave power). $V_{\mathrm{gl}}$ and $V_{\mathrm{gr}}$ are swept along the red arrow indicated in Fig. 1(c). The current at $\Delta E=0$ and $150 \mu \mathrm{eV}$ corresponds to resonant tunneling through the ground states (GG) and through the left ground state and an excited state in the right dot (GE1), respectively. A clear resonance is observed at $1.9446 \mathrm{GHz}(\Delta f=1.4 \mathrm{MHz})$, corresponding to the IDT resonance frequency. The inelastic current is due to absorption and emission of SAW phonons, as schematically depicted in the energy diagrams (b) and (d), respectively. The energy diagram for elastic resonant tunneling is shown in (c). 
explained by a propagating SAW in the adiabatic limit as follows. Energy diagrams for positive $\Delta E, \Delta E=0$, and negative $\Delta E$ are shown in Figs. 2(b)-2(d), respectively. Elastic current now appears at the time-dependent resonant condition $\Delta \tilde{E}(t)=0$. By assuming that the current is simply proportional to the time spent at resonant condition, current peaks appear at $\Delta E \pm \eta V_{\text {pe }}$ (i.e., at the classical turning points). The peak splitting is, hence, related to the piezoelectric potential amplitude.

The microwave power dependence of the current spectra is presented in Fig. 3(a). The peak splitting clearly increases with microwave power $P$. In Fig. 3(d), the splitting is plotted (black dots) as a function of the amplitude of the microwave voltage applied to the IDT $V_{\text {IDT }}$, confirming the linear dependence [10].

Since the tunneling rate (about $1 \mathrm{MHz}$ for $100 \mathrm{fA}$ current in our weakly coupled DQD) is much smaller than $f_{\text {SAW }}$, an electronic state in one dot acquires a phase, which is given by the integration of the oscillating potential, relative to another state in the other dot [11]. This nonadiabatic effect appears, for example, as photon assisted tunneling, as evidenced in various devices under microwave or farinfrared irradiation [8]. In our case, the oscillating potential is obviously induced by phonons. One can say that the DQD is exposed to surface acoustic phonons with energy $h f_{\text {SAW }}=8 \mu \mathrm{eV}$. The energy-dependent tunnel rate $\tilde{\Gamma}(E)$ from the left dot to the right dot in the presence of the phonon field is given by the same theory $[1,11]$

$$
\tilde{\Gamma}(\Delta E)=\sum_{n=-\infty}^{\infty} J_{n}^{2}(\alpha) \Gamma\left(\Delta E+n h f_{\mathrm{SAW}}\right)
$$

where $n=0, \pm 1, \pm 2, \ldots$ is the number of phonons involved in the absorption (positive $n$ ) and emission (negative $n), \Gamma(E)$ is the tunnel rate without phonons, and $J_{n}^{2}(\alpha)$ is the squared $n$th order Bessel function of the first kind evaluated at normalized amplitude $\alpha=e V_{\text {ac }} / h f_{\text {SAW }}$ [see inset in Fig. 3(c)]. The modulated DQD current $\tilde{I}$ then becomes [7]

$$
\tilde{I}=e\left|t_{12}\right|^{2} \Gamma_{R} \sum_{n=-\infty}^{\infty} \frac{J_{n}^{2}(\alpha)}{\Gamma_{R}^{2} / 4+\left(n 2 \pi f_{\mathrm{SAW}}-\Delta E / h\right)^{2}},
$$

where $\left|t_{12}\right|$ is the modulus of the tunnel coupling between the two dots, and $\Gamma_{R}$ is the tunnel rate from the right dot to the right lead. Inelastic current is allowed whenever the level spacing equals an integer number times the phonon energy, i.e., $\Delta E+n h f_{\mathrm{SAW}}=0$. The current thus consists of a number of satellite peaks, separated by the phonon energy $h f_{\text {SAW }}$. The Bessel function describes the probability that an electron absorbs $(n>0)$ or emits $(n<0) n$ phonons. It should be noted that Eq. (2) approaches the adiabatic limit for $\alpha \gg 1$.

Our DQD device has a resonant current linewidth of $14 \mu \mathrm{eV}$, even at zero microwave power, which is not sufficient to resolve a phonon sideband with spacing
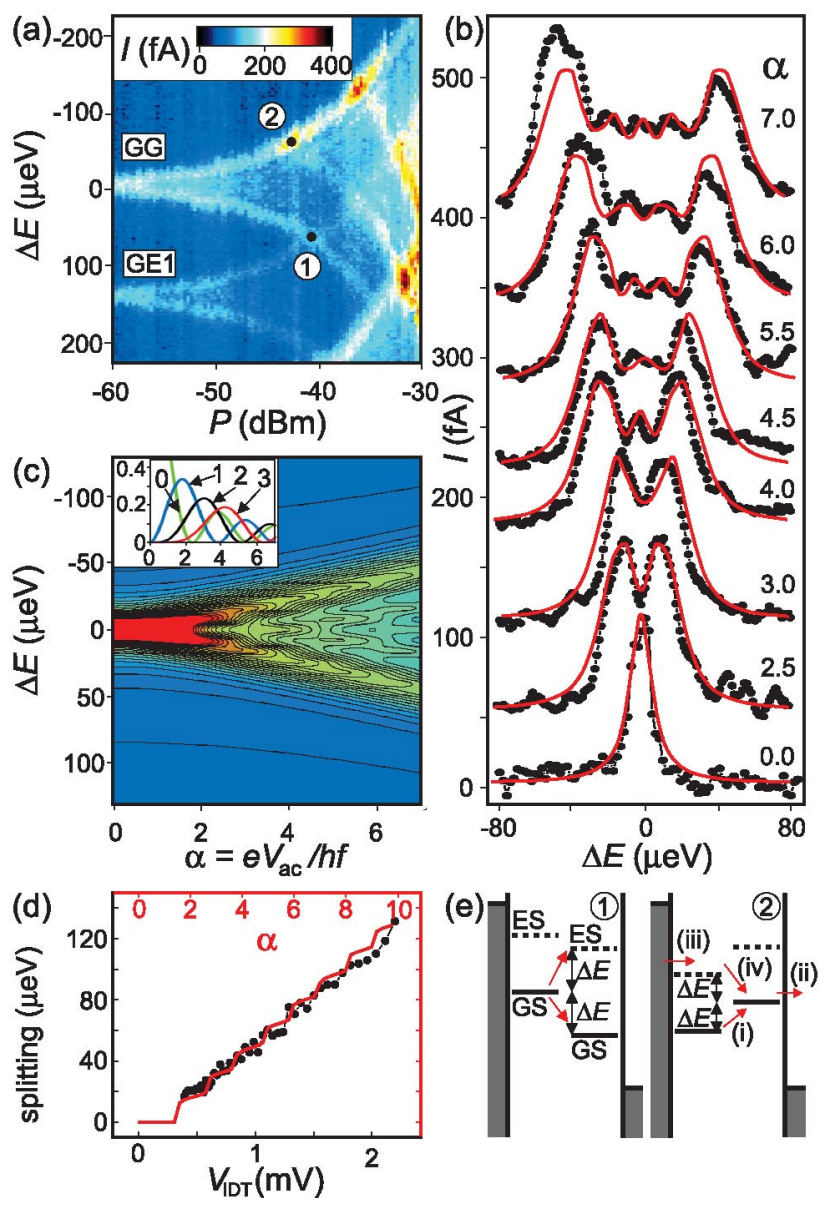

FIG. 3 (color). (a) Color scale plot of the DQD current versus $\Delta E$ and microwave power $P$, at $f_{\mathrm{SAW}}=1.9446 \mathrm{GHz}$, for the same transitions as in Fig. 2. (b) Experimental (black dots) and calculated (red curves) current spectra for different microwave powers, extracted from (a) and (c), respectively. The experimental microwave power incident on the IDT is converted to normalized potential amplitude $\alpha$ using (d). The current height of the calculated spectra is fitted to the experimental data. (c) Calculated DQD current versus $\Delta E$ and $\alpha$ in the nonadiabatic limit, as explained in the text. Inset: Squared Bessel functions $J_{n}^{2}(\alpha)$ for $n=0,1,2$, and 3. (d) Splitting of the current peaks as a function of the amplitude of the microwave voltage $V_{\text {IDT }}$ applied to the IDT for the experimental data (black data points and axes) and current peak splitting derived from the calculated spectra in (c) as a function of $\alpha$ (red curve and axes). By matching the experimental and calculated curves, the conversion between $P$ and $\alpha$ is found. (e) Schematic energy level diagrams for positions 1 and 2 indicated in (a). The transitions (i)-(iv) are discussed in the text.

$h f_{\text {SAW }}=8 \mu \mathrm{eV}$ [12]. However, we do observe clear evidence of nonadiabatic effects in the current spectra, as described below. Figure 3(b) shows the DQD current as function of $\Delta E$ for different $P$. The lowest curve is measured at zero power and represents the elastic current. The fit of the elastic current $I_{\mathrm{el}}$ (red curve) is a Lorentzian with a FWHM of $14 \mu \mathrm{eV}$. The expected current at finite microwave power $I_{\mathrm{SAW}}(\Delta E)$ is now derived from the zero- 
power curve as $I_{\mathrm{SAW}}(\Delta E)=\sum_{n=-\infty}^{\infty} J_{n}^{2}(\alpha) I_{\mathrm{el}}(\Delta E+$ $\left.n h f_{\text {SAW }}\right)$ and is plotted versus $\alpha$ in Fig. 3(c). For $\alpha \gtrsim 2$, the resonant current splits in two peaks whose positions approach $\Delta E= \pm \alpha h f_{\mathrm{SAW}}$, corresponding to the adiabatic limit. The splitting between the calculated current peaks versus $\alpha$ is plotted (red solid curve) together with the experimental splitting versus $V_{\text {IDT }}$ in Fig. 3(d). Very good agreement between the experimental data and the nonadiabatic calculation is found when we relate $V_{\text {IDT }}$ to the normalized ac potential $\alpha$ according to $\alpha=$ $0.09 \eta e V_{\mathrm{IDT}} / h f$. The first factor, corresponding to the loss in the IDT, is in good agreement with the loss estimated from Fig. 1(b). The nonadiabatic calculation in Fig. 3 shows clear additional structure in between the split peaks. This structure originates from the phonon satellite peaks that should be individually resolvable at $\Delta E=n h f_{\mathrm{SAW}}$ if the peak width is smaller than the phonon energy. In our case, however, the peak width exceeds $h f_{\text {SAW }}$ (but is less than $\left.2 h f_{\mathrm{SAW}}\right)$. We actually find good agreement between the calculated current spectra and the experimental data (including the interpeak fine structure) at finite microwave power as shown in Fig. 3(b), where we have applied the $\alpha-P[\mathrm{dBm}]$ conversion derived in Fig. 3(d). Our data thus reveal clear quantum behavior, even when we cannot resolve individual phonon satellites.

Quantum behavior is also observed in multiple excitation processes between excited states at higher power. As indicated by (1)in Fig. 3(a), one of the split peaks of the GG resonance and one of the GE1 resonance touch around $-40 \mathrm{dBm}$, where the GS-GS level spacing and the spacing between the GS in the left dot and the first ES in the right dot both equal $V_{\mathrm{ac}}$, as shown in the left diagram in Fig. 3(e). At this condition, two phonon assisted tunneling processes (red arrows) are allowed from the GS of the left dot. There is another peak emerging for $P>-42 \mathrm{dBm}$ indicated by (2)in Fig. 3(a). This peak is associated with phonon assisted tunneling from an ES in the left dot to the GS of the right dot [right diagram in Fig. 3(e)]. This tunneling process is possible only if an electron (i) tunnels from the GS of the left dot to the GS of the right dot and (ii) escapes to the right lead, (iii) another electron tunnels into the ES of the left dot, (iv) followed by tunneling to the GS of the right dot under phonon emission. This explanation is consistent with the absence of the peak at lower power, where the GS in the left dot is permanently occupied. At higher power, more resonant peaks are resolved, which may be useful in analyzing the energy spectrum of our DQD.

Finally, we comment on the measurement sensitivity to the piezoelectric potential in our experiment. As discussed above, the current spectra reflect the amplitude of the local piezoelectric potential. The lowest power at which we can resolve peak splitting is $-58 \mathrm{dBm}$, corresponding to $V_{\mathrm{pe}}=$
$24 \mu \mathrm{V}$, which is several orders of magnitude smaller than the power used to induce dynamical quantum dots [6] and to induce lattice displacements measurable by optical interferometry [13]. The minimum detection power can be improved further by adjusting the DQD parameters. When the elastic current peak width is made smaller than the phonon energy, the piezoelectric potential can be derived from the amplitude of the phonon assisted tunneling current via the Bessel function dependence even for $\alpha \ll 1$. This may enable the measurement of lattice distortion due to vacuum fluctuations.

In conclusion, we have observed inelastic tunneling in a DQD two-level system coupled to a monochromatic SAW source. The transport through the DQD is well described by nonadiabatic Tien-Gordon theory for resonant tunneling between two discrete states with a time-dependent potential. We find that the DQD can be employed as a very sensitive SAW detector and is promising for studying electron-phonon interaction.

We thank S. Tarucha, P. V. Santos, R. Aguado, L.P. Kouwenhoven, and Y. Hirayama for fruitful discussions and help. We acknowledge financial support from DARPA Grant No. DAAD19-01-1-0659 of the QuIST program and SCOPE from the Ministry of Internal Affairs and Communications of Japan.

[1] W. G. van der Wiel et al., Rev. Mod. Phys. 75, 1 (2003).

[2] A. J. Leggett et al., Rev. Mod. Phys. 59, 1 (1987).

[3] T. Fujisawa et al., Science 282, 932 (1998); T. Fujisawa, W. G. van der Wiel, and L.P. Kouwenhoven, Physica (Amsterdam) 7E, 413 (2000).

[4] T. Brandes and B. Kramer, Phys. Rev. Lett. 83, 3021 (1999).

[5] A. A. Oliner, Acoustic Surface Waves (Springer-Verlag, Berlin, 1978).

[6] C.H.W. Barnes et al., Phys. Rev. B 62, 8410 (2000); J. A. H. Stotz et al., Nat. Mater. 4, 585 (2005).

[7] T. H. Stoof and Yu. V. Nazarov, Phys. Rev. B 53, 1050 (1996).

[8] W. G. van der Wiel et al., in Photon Assisted Tunneling in Quantum Dots in Strongly Correlated Fermions and Bosons in Low-Dimensional Disordered Systems, edited by I. V. Lerner et al. (Kluwer, New York, 2002), pp. 43-68.

[9] P. V. Santos (private communication).

[10] $V_{\mathrm{IDT}}=\sqrt{Z P}$, where $Z=50 \Omega$ and $P=10^{-3} 10^{P[\mathrm{dBm}] / 10}$.

[11] P. K. Tien and J. R. Gordon, Phys. Rev. 129, 647 (1963).

[12] This width is most probably due to charge instability in the heterostructure we used; see, e.g., M. Pioro-Ladrière et al., Phys. Rev. B 72, 115331 (2005).

[13] M. M. de Lima et al., J. Appl. Phys. 94, 7848 (2003). 